\title{
A Novel Autofocusing Approach for Estimating Directions-of-Arrival of Wideband Signals
}

\author{
Piya Pal and P. P. Vaidyanathan \\ Dept. of Electrical Engineering, MC 136-93 \\ California Institute of Technology, Pasadena, CA 91125, USA \\ E-mail: piyapal@caltech.edu,ppvnath@systems.caltech.edu
}

\begin{abstract}
We propose a novel approach to the design of focusing matrices which play important role in the coherent methods for wideband direction-of-arrival estimation. We call this 'autofocusing' because unlike the conventional methods, our technique constructs the focusing matrices entirely by processing the received signal and does not require any preliminary DOA estimates. In this way, it overcomes the major drawback of the coherent methods which otherwise possess many desirable properties. Through computer simulations, the proposed method is found to exhibit satisfactory performance in comparison to the existing wideband DOA estimation algorithms.
\end{abstract}

Index Terms - Direction-of-arrival (DOA), Wideband Sources, Focusing matrix, Signal subspace method.

\section{INTRODUCTION}

The problem of estimating the directions-of-arrival (DOA) of signals impinging on a sensor array has widespread application in source localization for radar, sonar and wireless communication systems. When the incoming signals are wideband, the most popular approach is to decompose the wideband signal into narrowband components and wideband DOA estimation in that case is mainly concerned with finding a clever way to use the multiple correlation matrices at different frequencies to get accurate DOA estimates.

The incoherent signal subspace method (ISSM) [8] is the simplest wideband method which estimates the source DOAs separately at each narrowband frequency and then constructs the final estimate by taking an average. While this works well at high SNR, the performance can suffer severely at low SNR because even a single outlier from one narrowband component can potentially lead to inaccurate estimates through the averaging process. Also it is incapable of handling coherent sources. To overcome these drawbacks, several coherent methods have been suggested [1]-[4], [7]. Of these, the coherent signal subspace method (CSSM) [7] was the earliest. It aimed at focusing the signal subspace at each frequency to that at a certain reference focusing frequency and then constructing a single correlation matrix by summing over these focused correlation matrices, on which narrowband DOA estimation algorithms could be applied. This method has good performance at low SNR and can also handle coherent sources [7]. However, construction of the focusing matrices needs preliminary estimates of DOAs and the performance of CSSM is sensitive to the error in the preliminary estimates, which can give rise to biased final estimates [6]. The weighted average of signal subspaces (WAVES) [1] is another coherent method and it also requires focusing matrices. Though it can avoid initial estimates by using BI-CSSM technique, its performance

\footnotetext{
${ }^{1}$ Work supported in parts by the ONR grant N00014-08-1-0709, and the
} California Institute of Technology. is worse than when it uses focusing matrices with good initial estimates. Recently, a novel wideband DOA algorithm called test of orthogonality of projected subspaces (TOPS) [9] has been proposed. It is in between the coherent and incoherent processing, performing best at low to mid SNR and does not require initial estimates. However, the spatial spectrum used in TOPS can show false peaks which is a disadvantage of this method.

It is seen that coherent methods are definitely advantageous in terms of good performance at low SNR and capability for handling coherent sources. However, the dependence of the focusing matrices used in coherent methods on the preliminary DOA estimates, is a severe drawback of these methods which gives rise to increased computation and also potentially biased estimates. To overcome this problem, in this paper, we suggest a new technique to focus the signal subspaces at different frequencies, which does not require the preliminary DOA estimates. This technique, which we call autofocusing, constructs the focusing matrices entirely on the basis of the received data and does not require knowledge of array manifold. Hence careful calibration of the array is not needed in their construction. Also, the focusing matrices are made unitary by construction so that there is no focusing loss.

The rest of the paper is organized as follows. In Section II, we briefly review the major wideband DOA estimation algorithms. In Section III, we discuss the proposed method. In Section IV, we provide numerical examples to compare the proposed method with existing ones and finally Section V concludes the paper.

\section{BACKGROUND REVIEW}

\section{A. Signal Model}

Consider an $N$ element linear array with unambiguous array manifold, i.e., $D(\leq N)$ steering vectors of the array corresponding to $D$ different source angles are linearly independent, spanning a $D$ dimensional subspace, also known as the signal subspace. Consider $D(<N)$ wideband sources $s_{1}(t), s_{2}(t), \cdots, s_{D}(t)$ impinging on the array from different directions of arrival $\theta_{1}, \theta_{2}, \cdots, \theta_{D}$ respectively. The signal received at the $n$th sensor is given by

$$
\begin{gathered}
x_{n}(t)=\sum_{d=1}^{D} s_{d}\left(t-\tau_{n}\left(\theta_{d}\right)\right)+\eta_{n}(t) \\
n=1,2, \cdots, N
\end{gathered}
$$

where $\tau_{n}\left(\theta_{d}\right)$ is the propagation delay associated with the $d$ th source and the $n$th sensor and $\eta_{n}($.$) is the additive noise at$ the $n$th sensor. The incoming signal at each sensor is first 
sampled at frequency $f_{s}$ and the samples are then partitioned into segments of $K=\Delta T f_{s}$ samples each. Then, a $K$-point DFT is applied to the $K$ samples in a segment. The DFT coefficients from the $N$ sensors can be expressed as

$$
\mathbf{X}[i]=\mathbf{A}\left(f_{i}\right) \mathbf{S}[i]+\mathbf{N}[i], \quad i=0,1, \cdots, K-1
$$

where

$$
f_{i}=\frac{i}{K} f_{s}
$$

and $\mathbf{X}[i]=\left[\begin{array}{llll}X_{1}[i] & X_{2}[i] & \cdots & X_{N}[i]\end{array}\right]^{T}$ with $X_{n}[i]$ denoting the $i$ th DFT coefficient of samples of $x_{n}(t)$. Here,

$$
\mathbf{A}\left(f_{i}\right)=\left[\begin{array}{llll}
\mathbf{a}_{\theta_{1}}\left(f_{i}\right) & \mathbf{a}_{\theta_{2}}\left(f_{i}\right) & \cdots & \mathbf{a}_{\theta_{D}}\left(f_{i}\right)
\end{array}\right],
$$

with

$$
\mathbf{a}_{\theta_{d}}\left(f_{i}\right)=\left[e^{-j 2 \pi f_{i} \tau_{1}\left(\theta_{d}\right)}, \cdots, e^{-j 2 \pi f_{i} \tau_{N}\left(\theta_{d}\right)}\right]^{T}
$$

as the steering vector of the array at frequency $f_{i}$ for the $d$ th source. In (2),

$$
\begin{gathered}
\mathbf{N}[j]=\left[\begin{array}{llll}
N_{1}[i] & N_{2}[i] & \cdots & N_{N}[i]
\end{array}\right]^{T}, \\
\mathbf{S}[i]=\left[\begin{array}{llll}
S_{1}[i] & S_{2}[i] & \cdots & S_{D}[i]
\end{array}\right]^{T}
\end{gathered}
$$

where $N_{n}[i]$ and $S_{d}[i]$ are the $i$ th DFT coefficients of samples of $\eta_{n}(t)$ and $s_{d}(t)$ respectively. Under the assumption that $\Delta T$ is long enough compared to the correlation time of the signals and the noise so that we can regard the DFT coefficients uncorrelated [2], [7], we can write

$$
\begin{aligned}
\mathbf{R}_{x x}[i] & \triangleq E\left[\mathbf{X}[i] \mathbf{X}^{H}[i]\right] \\
& =\mathbf{A}\left(f_{i}\right) \mathbf{R}_{s s}[i] \mathbf{A}^{H}\left(f_{i}\right)+\sigma_{n}^{2} \mathbf{I},
\end{aligned}
$$

where $\mathbf{R}_{s s}[i]=E\left[\mathbf{S}[i] \mathbf{S}^{H}[i]\right]$ and we have assumed the noise samples to be zero mean temporally and spatially white Gaussian random process with variance $\sigma_{n}^{2}$ so that the autocorrelation matrix at each DFT frequency is $\sigma_{n}^{2} \mathbf{I}$. Assuming that the $D$ sources are uncorrelated, the $D \times D$ matrix $\mathbf{R}_{s s}[i]$ has full rank. Then, if the eigenvectors of $\mathbf{R}_{x x}[i]$ are ordered in decreasing order with respect to their eigenvalues, the first $D$ eigenvectors span the same subspace of dimension $D$ (known as signal subspace at frequency $\left.f_{i}\right)$ as $\mathcal{R}\left(\mathbf{A}\left(f_{i}\right)\right.$ ) (range space of $\left.\mathbf{A}\left(f_{i}\right)\right)$ and are known as the signal subspace eigenvectors. The last $N-D$ eigenvectors are called the noise subspace eigenvectors and they span a subspace of dimension $N-D$ orthogonal to the signal subspace.

\section{B. Review of Coherent and Incoherent Methods of Wideband DOA Estimation}

The incoherent signal subspace method (ISSM) [8] is the simplest wideband DOA estimation method which estimates the signal and noise subspace at each frequency independently and then the estimated DOAs from each frequency bin are averaged in some way to get the final estimate. This method, as mentioned in the earlier section, suffers at low SNR [7]. The coherent method [7] attempts at solving this problem by combining the signal subspaces coherently into one single autocorrelation matrix to which narrowband high resolution methods (such as MUSIC) can be applied. The key idea is to design a focusing matrix $\mathbf{T}\left(f_{i}\right)$ which transforms the array manifold at frequency $f_{i}$ to that at a common frequency $f_{0}$ as follows

$$
\mathbf{T}\left(f_{i}\right) \mathbf{A}\left(f_{i}\right)=\mathbf{A}\left(f_{0}\right) .
$$

Then the coherent autocorrelation matrix is generated as

$$
\begin{aligned}
\mathbf{R}_{c o h}= & \sum_{i=0}^{K-1} \mathbf{T}\left(f_{i}\right) \mathbf{R}_{x x}[i] \mathbf{T}^{H}\left(f_{i}\right) \\
& =\mathbf{A}\left(f_{0}\right) \mathbf{R}_{s} \mathbf{A}^{H}\left(f_{0}\right)+\sigma_{n}^{2} \sum_{i=0}^{K-1} \mathbf{T}\left(f_{i}\right) \mathbf{T}^{H}\left(f_{i}\right) .
\end{aligned}
$$

where $\mathbf{R}_{s}=\sum_{i=0}^{K-1} \mathbf{R}_{s s}[i]$. Now any standard narrowband DOA estimation method such as MUSIC [5], can be applied by computing the eigenvectors of the matrix pencil $\left(\mathbf{R}_{c o h}, \sum_{i=0}^{K-1} \mathbf{T}\left(f_{i}\right) \mathbf{T}^{H}\left(f_{i}\right)\right)$. The coherent method is potentially capable of better performance even at low SNR as it first focuses the energy at different frequencies at the focusing frequency and then performs narrowband DOA estimation at that focusing frequency. Also by the frequency averaging process, it can handle correlated sources. WAVES [1] is another interesting coherent method which, instead of using the entire autocorrelation matrix at different frequencies, uses sum of suitably weighted signal subspaces. However, it also requires focusing matrices.

The focusing matrices are non-unique and several discussions on construction of these focusing matrices can be found in [2], [3], [7]. The most popular way to generate these focusing matrices is to construct a unitary focusing matrix by minimizing the Frobenius norm of the manifold mismatches, viz.,

$$
\begin{gathered}
\min _{\mathbf{T}\left(f_{i}\right)}\left\|\mathbf{A}\left(f_{0}\right)-\mathbf{T}\left(f_{i}\right) \mathbf{A}\left(f_{i}\right)\right\|_{F} \\
\left.\quad \text { subject to } \mathbf{T}\left(f_{i}\right) \mathbf{T}^{H}\left(f_{i}\right)\right)=\mathbf{I} .
\end{gathered}
$$

This class of focusing matrices is called RSS (rotational signalsubspace) matrices [3] and the matrix is required to be unitary in order to preserve the SNR before and after focusing. The focusing matrices are further discussed in [2] where it is shown that the focusing matrices (including the RSS matrices) belong to the broader class of matrices called SST (signal-subspace transformation) matrices.

However, to construct the focusing matrices following any of the popular methods, it is crucial to have some preliminary estimate of the DOAs. Poor initial estimates might lead to inaccurate focusing and give rise to final DOA estimates which are biased [6]. The need for initial DOA estimates can be avoided by the BI-CSSM method [4] which utilizes a set of frequency invariant beams spanning the field-of-view (FOV). However the performance of this method heavily depends on the size of the FOV and the array geometry.

\section{AUTOFOCUSING APPROACH TO COHERENT SIGNAL SUBSPACE BASED WIDEBAND DOA ESTIMATION}

In this paper, we propose a novel technique of generating focusing matrices, which do not require any preliminary DOA estimation, and yet can coherently combine the signal subspaces at different frequencies into one coherent subspace at the chosen frequency, thereby assuring all the advantages offered by CSS method. The need for preliminary DOA 
estimates is avoided by noticing the simple fact that the signal subspace eigenvectors span the same subspace as the array manifold at each frequency and so, we can use them to form the focusing matrix, instead of using the array steering vectors, which require the preliminary knowledge of the DOAs.

Let us consider the same signal model as described in Section I. In particular, the autocorrelation matrix for the $i$ th DFT coefficient is

$$
\mathbf{R}_{x x}[i]=\mathbf{A}\left(f_{i}\right) \mathbf{R}_{s s}[i] \mathbf{A}^{H}\left(f_{i}\right)+\sigma_{n}^{2} \mathbf{I} .
$$

Suppose, we want to focus to a frequency $f_{0}$. Then the focusing matrix proposed by our method is

$$
\mathbf{T}_{\text {auto }}\left(f_{i}\right)=\frac{1}{\sqrt{K}} \mathbf{U}\left(f_{0}\right) \mathbf{U}^{H}\left(f_{i}\right)
$$

where $K$ is the number of frequency bins considered and $\mathbf{U}\left(f_{i}\right)$ is a $N \times N$ unitary matrix whose columns are the eigenvectors of the autocorrelation matrix $\mathbf{R}_{x x}[i]$. We assume that all sources have non zero energy at all frequencies $\left\{f_{i}\right\}$ under consideration. This choice of focusing matrix, as we shall see shortly, will satisfy the purpose we discussed in the previous paragraph. It is to be noted that this matrix belongs to the same class of SST matrices as proposed by Doron in [2]. Since the eigenvectors of $\mathbf{R}_{x x}$ [i] can be grouped into signal space and noise space eigenvectors, we can represent $\mathbf{U}\left(f_{i}\right)$ as

$$
\mathbf{U}\left(f_{i}\right)=\left[\begin{array}{ll}
\mathbf{U}_{s s}\left(f_{i}\right) & \mathbf{U}_{N}\left(f_{i}\right)
\end{array}\right]
$$

where $\mathbf{U}_{s s}\left(f_{i}\right)$ is a $N \times D$ matrix whose columns represent the $D$ orthonormal eigenvectors of $\mathbf{R}_{x x}[i]$ corresponding to the $D$ largest eigenvalues, and $\mathbf{U}_{N}\left(f_{i}\right)$ is a $N \times(N-D)$ matrix whose columns represent the remaining $(N-D)$ orthonormal eigenvectors of $\mathbf{R}_{x x}[i]$. It is to be noted that to construct $\mathbf{T}_{\text {auto }}\left(f_{i}\right)$, we do not need to know the number of sources, $D$, since we are using the complete set of eigenvectors of $\mathbf{R}_{x x}[i]$ as columns of $\mathbf{U}\left(f_{i}\right)$, as opposed to using, say, $D$ of them. However this does not prevent us from representing $\mathbf{U}\left(f_{i}\right)$ as in (16) because we can always construct $\mathbf{U}\left(f_{i}\right)$ by making the $j$ th column equal to the eigenvector corresponding to the $j$ th largest eigenvalue. We can now multiply $\mathbf{X}[i]$ by the focusing matrix and define the transformed vector

$$
\mathbf{Y}[i]=\mathbf{T}_{\text {auto }}\left(f_{i}\right) \mathbf{X}[i] .
$$

Taking the autocorrelation of $\mathbf{Y}[i]$, substituting (14) and (15), and using the unitarity of $\mathbf{T}_{\text {auto }}\left(f_{i}\right)$, we get,

$$
\begin{aligned}
\mathbf{R}_{y y}[i]= & E\left[\mathbf{Y}[i] \mathbf{Y}[i]^{H}\right] \\
= & \mathbf{T}_{\text {auto }}\left(f_{i}\right) \mathbf{A}\left(f_{i}\right) \mathbf{R}_{s s}[i] \mathbf{A}^{H}\left(f_{i}\right) \mathbf{T}_{\text {auto }}^{H}\left(f_{i}\right) \\
& +\frac{1}{K} \sigma_{n}^{2} \mathbf{I}
\end{aligned}
$$

Now we have,

$$
\begin{aligned}
& \mathbf{T}_{\text {auto }}\left(f_{i}\right) \mathbf{A}\left(f_{i}\right) \\
& =\frac{1}{\sqrt{K}}\left[\begin{array}{ll}
\mathbf{U}_{s s}\left(f_{0}\right) & \mathbf{U}_{N}\left(f_{0}\right)
\end{array}\right]\left[\begin{array}{c}
\mathbf{U}_{s s}^{H}\left(f_{i}\right) \\
\mathbf{U}_{N}^{H}\left(f_{i}\right)
\end{array}\right] \mathbf{A}\left(f_{i}\right) \\
& =\frac{1}{\sqrt{K}}\left[\begin{array}{ll}
\mathbf{U}_{s s}\left(f_{0}\right) & \mathbf{U}_{N}\left(f_{0}\right)
\end{array}\right]\left[\begin{array}{c}
\mathbf{U}_{s s}^{H}\left(f_{i}\right) \mathbf{A}\left(f_{i}\right) \\
0
\end{array}\right] \\
& =\frac{1}{\sqrt{K}} \mathbf{U}_{s s}\left(f_{0}\right) \mathbf{U}_{s s}^{H}\left(f_{i}\right) \mathbf{A}\left(f_{i}\right)
\end{aligned}
$$

where (19) is due to the fact that the noise eigenvectors at frequency $f_{i}$ are orthogonal to the array manifold $\mathbf{A}\left(f_{i}\right)$ at frequency $f_{i}$. Substituting (20) into (18), we get,

$$
\mathbf{R}_{y y}[i]=\frac{1}{K} \mathbf{U}_{s s}\left(f_{0}\right) \tilde{\mathbf{R}}[i] \mathbf{U}_{s s}^{H}\left(f_{0}\right)+\frac{1}{K} \sigma_{n}^{2} \mathbf{I}
$$

where

$$
\tilde{\mathbf{R}}[i]=\mathbf{U}_{s s}^{H}\left(f_{i}\right) \mathbf{A}\left(f_{i}\right) \mathbf{R}_{s s}[i] \mathbf{A}^{H}\left(f_{i}\right) \mathbf{U}_{s s}\left(f_{i}\right) .
$$

We next sum (21) corresponding to the $K$ frequencies to get the coherently combined autocorrelation matrix as

$$
\begin{aligned}
\mathbf{R}_{c o h} & =\sum_{i=0}^{K-1} \mathbf{R}_{y y}[i] \\
& =\frac{1}{K} \mathbf{U}_{s s}\left(f_{0}\right)\left(\sum_{i=0}^{K-1} \tilde{\mathbf{R}}[i]\right) \mathbf{U}_{s s}^{H}\left(f_{0}\right)+\sigma_{n}^{2} \mathbf{I} \\
& =\mathbf{U}_{s s}\left(f_{0}\right) \mathbf{R}_{s} \mathbf{U}_{s s}^{H}\left(f_{0}\right)+\sigma_{n}^{2} \mathbf{I}
\end{aligned}
$$

where

$$
\mathbf{R}_{s}=\frac{1}{K} \sum_{i=0}^{K-1} \tilde{\mathbf{R}}[i]=\frac{1}{K} \sum_{i=0}^{K-1} \mathbf{U}_{s s}^{H}\left(f_{i}\right) \mathbf{A}\left(f_{i}\right) \mathbf{R}_{s s}[i] \mathbf{A}^{H}\left(f_{i}\right) \mathbf{U}_{s s}\left(f_{i}\right) .
$$

$\mathbf{R}_{\text {coh }}$ plays the role of the universal focused sample correlation matrix, where the signal subspaces at different frequencies are focused to the signal subspace at $f_{0}$ which is spanned by the columns of $\mathbf{U}_{s s}\left(f_{0}\right)$. It can be seen that (25) has similar form as (12). However, instead of the actual array manifold $\mathbf{A}\left(f_{0}\right)$ as the focusing array manifold, we have the matrix $\mathbf{U}_{s s}\left(f_{0}\right)$ whose columns span the same subspace as $\mathbf{A}\left(f_{0}\right)$ and hence, as we shall show shortly, they carry the same information about the focusing signal subspace. This is the primary reason why, in our proposed method, the focusing can be performed without the preliminary DOA estimates. The signal autocorrelation matrices at different frequencies are transformed, and then summed to get the total correlation matrix $\mathbf{R}_{s}$ whose rank will be discussed in the following theorem. As the focusing matrices are unitary, the spatial whiteness of the noise is preserved and hence the noise autocorrelation matrix remains unchanged. Thus, (25) has the form of a narrowband sample autocorrelation matrix from which we can extract the DOA estimates following similar technique as narrowband MUSIC algorithm, as explained by the following theorem.

Lemma: $\mathbf{R}_{s}$ as given by (26), is a non negative matrix satisfying

$$
\max _{i}\left\{\operatorname{rank}\left(\mathbf{R}_{s s}[i]\right)\right\} \leq \operatorname{rank}\left(\mathbf{R}_{s}\right) \leq D .
$$

If the sources are uncorrelated, then $\mathbf{R}_{s}$ is a positive definite matrix with

$$
\operatorname{rank}\left(\mathbf{R}_{s}\right)=D .
$$

Proof: In (22), since $\mathbf{U}_{s s}\left(f_{i}\right)$ and $\mathbf{A}\left(f_{i}\right)$ are $N \times D$ matrices with full column rank, spanning the same range space, $\mathbf{U}_{s s}^{H}\left(f_{i}\right) \mathbf{A}\left(f_{i}\right)$ is a full-rank $D \times D$ matrix and so

$$
\operatorname{rank}(\tilde{\mathbf{R}}[i])=\operatorname{rank}\left(\mathbf{R}_{s s}[i]\right)
$$


Moreover, as $\mathbf{R}_{s s}[i]$ is non negative definite (allowing coherent sources), so must be $\tilde{\mathbf{R}}[i]$. Therefore $\mathbf{R}_{s}$ is itself non negative definite. Also, it is easy to check that

$$
\mathcal{N}\left(\mathbf{R}_{s}\right)=\bigcap_{i=0}^{K-1} \mathcal{N}(\tilde{\mathbf{R}}[i])
$$

and hence, rank of the $D \times D$ matrix $\mathbf{R}_{s}$ satisfies (27).

When the sources are uncorrelated, then $\mathbf{R}_{s s}[i]$ is positive definite for $i=0,1, \cdots, K-1$. So, using (29), we can say that $\operatorname{rank}(\tilde{\mathbf{R}}[i])=D \forall i$. This, along with (27), implies that $\operatorname{rank}\left(\mathbf{R}_{s}\right)=D$ and hence $R_{s}$ becomes a full rank positive definite matrix.

Theorem 1: Let $\mathbf{R}_{\text {coh }}$ be the coherently combined sample autocorrelation matrix of the sensor outputs as given by (25). Let $\mathbf{e}_{i}$ and $\lambda_{i}, i=1,2, \cdots, N$ be the eigenvectors and the corresponding eigenvalues, (in decreasing order), of $\mathbf{R}_{c o h}$. Then, we have

(a) $\lambda_{D+1}=\lambda_{D+2}=\cdots=\lambda_{N}=\sigma_{n}^{2}$

(b) Assuming the sources are uncorrelated, column span of $\mathbf{E}_{N} \triangleq\left[\begin{array}{llll}\mathbf{e}_{D+1} & \mathbf{e}_{D+2} & \cdots & \mathbf{e}_{N}\end{array}\right]$ is orthogonal to Range space of $\mathbf{A}\left(f_{0}\right)$.

Proof: When the sources are uncorrelated, we know from (28), that $\mathbf{R}_{s}$ is a positive definite matrix. Since $\mathbf{U}_{s s}\left(f_{0}\right)$ is $N \times D$ matrix with full column rank $D$, so $\left.\mathbf{U}_{s s}\left(f_{0}\right) \mathbf{R}_{s} \mathbf{U}_{s s}^{H}\left(f_{0}\right)\right)$ is a non negative definite rank- $D$ matrix, whose columns span the same subspace as $\mathcal{R}\left(\mathbf{A}\left(f_{0}\right)\right)$ since $\mathcal{R}\left(\mathbf{U}_{s s}\left(f_{0}\right)\right)=\mathcal{R}\left(\mathbf{A}\left(f_{0}\right)\right)$. The eigenstructure of $\mathbf{R}_{c o h}$ in that case is exactly the same as that of the sample autocorrelation matrix encountered in narrowband MUSIC algorithm and hence the proof of this part of the theorem follows the same lines as given in [5].

The theorem ensures that we can perform narrowband MUSIC on the coherently combined covariance matrix obtained by using our proposed focusing matrix. We have explicitly proved that all $D$ sources can be identified when they are uncorrelated. However, this condition can be relaxed to include even correlated sources. In this case, though the source covariance matrices $\mathbf{R}_{s s}[i]$ are singular, the singularity may be removed by the frequency averaging process so that the averaged matrix $\mathbf{R}_{s}$ becomes non singular.

\section{Advantages of the proposed autofocusing technique:}

We now summarize the advantages of our proposed focusing method:

(i) It does not need any initial estimate of the DOAs to construct focusing matrix. Thus it completely avoids the need for preprocessing.

(ii) The focusing matrix $\mathbf{T}_{\text {auto }}\left(f_{i}\right)$ is a unitary matrix by construction, and hence, according to [2], [3], it results in no focusing loss. Also, it preserves the spatial whiteness of the noise, so that the coherently combined noise autocorrelation matrix remains diagonal.

(iii) Construction of the focusing matrix at a particular frequency does not require the knowledge of the number of sources since we use the full set of eigenvectors (as opposed to using only the signal-subspace eigenvectors) of the autocorrelation matrix at that frequency. This in turn avoids the errors that might occur due to wrong detection of number of sources at low SNR locally at a given frequency.

(iv) The focusing matrices are constructed entirely on the basis of the received data, by the eigendecomposition of the signal autocorrelation matrix at different frequencies. So it can be viewed as a fully data dependent method which can be adapted to the changing scenario.

(v) Since we do not use the array manifold explicitly in constructing the focusing matrices, it does not require accurate calibration and can avoid the errors that occur due to mismatches. The only time the array needs to be calibrated is when we apply MUSIC to the coherently combined autocorrelation matrix. However, if we use ESPRIT instead of MUSIC, even this calibration can be avoided.

\section{Simulation Examples}

In this section, the performance of the proposed method is evaluated and compared against popular wideband DOA estimation algorithms through simulation examples. We consider $D=3$ broadband sources impinging on a 10 element ULA from $0^{\circ}, 30^{\circ}$ and $45^{\circ}$. The sampling frequency is chosen to be twice the highest frequency. The sources occupy the frequency band between $\pi / 2$ and $\pi$ in the digital frequency domain. The sensor spacing is chosen to be $\lambda_{\min } / 2$ where $\lambda_{\min }$ is the wavelength corresponding to the highest frequency in the signal. This spacing ensures that there is no aliasing in the spatial domain.

The samples of the impinging signal are divided into $M=$ 100 segments of $K=256$ samples each. In each segment the 256 samples are converted to frequency domain by a 256-point DFT which are then processed using four different algorithms: i) The proposed autofocusing method. ii) CSSM using RSS focusing matrices [3], [7] with perfect intial DOA estimates (named CSSM I) iii) CSSM using RSS focusing matrices with intial DOA estimates obatined by delay-andsum beamforming (named CSSM II), and iv) the recently proposed TOPS [9] algorithm. The RSS focusing matrices are constructed following [7] and the focusing frequency $f_{0}$ for both the CSSM methods and the proposed method is chosen to be the middle frequency of the band.

The performances of the four algorithms are compared for the source at $45^{\circ}$ in terms of their RMSE in Fig. 1 and bias in Fig. 2. The proposed autofocusing technique is found to outperform CSSM II and TOPS with respect to both RMSE and bias. The CSSM method with perfect initial DOA estimates exhibits the best performance but it is impractical. It is to be noted that CSSM II, which is the more practical way to implement CSSM, suffers from bias even at high SNR which is due to inaccurate initial DOA estimates. The proposed method, however, not requiring any such initial estimates, gives rise to unbiased estimates as evident from the plots.

It is also to be noted that TOPS spectrum can show spurious peaks at angles other than the true DOAs. To exhibit this, the TOPS spectrum (inverse of the smallest singular value) and the MUSIC spectrum for the proposed method are plotted (as a function of angle of arrival) at $\mathrm{SNR}=0 \mathrm{~dB}$. While the proposed method clearly shows only three peaks, the TOPS spectrum shows several other false peaks, which can potentially get erroneously detected as source DOAs. 


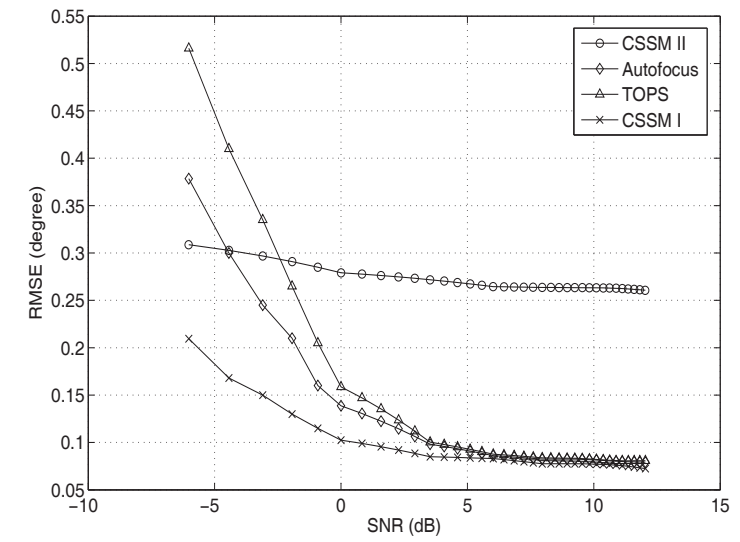

Fig. 1. Comparison of RMSE of the different wideband DOA estimation algorithms v/s SNR for the source at $45^{\circ}$.

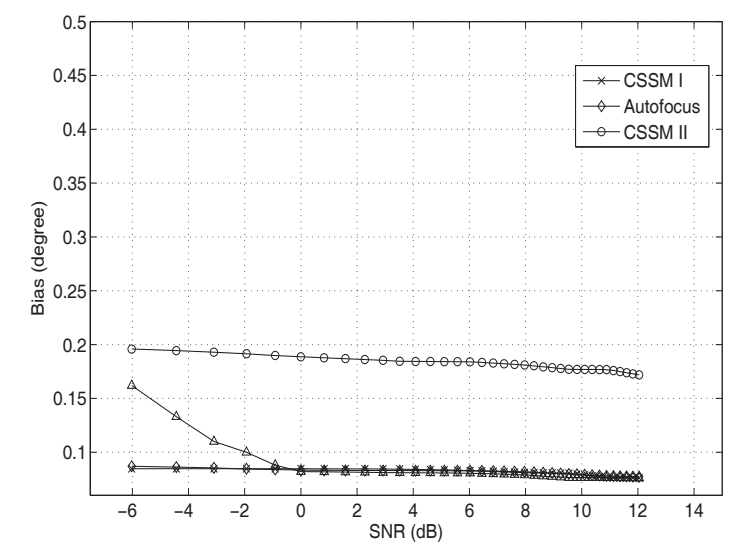

Fig. 2. Comparison of bias of the different wideband DOA estimation algorithms v/s SNR for the source at $45^{\circ}$.

\section{CONCLUSION}

In this paper, we proposed a new wideband DOA estimation algorithm based on the coherent signal subspace approach. It provides a solution to one of the major drawbacks of coherent methods, viz., the requirement of initial DOA estimates, while exhibiting the desirable properties of coherent methods. The focusing matrices proposed by our method are constructed in a fully data-dependent manner, directly from the received data, thereby avoiding the need for careful array calibration. Also it results in no SNR loss as the matrices are unitary by construction. However, the performance of our method is likely to depend on the choice of the focusing frequency (subspace) and future work will concentrate on the selection of the best focusing frequency based on the received data. It will also be interesting to perform asymptotic performance analysis of the proposed algorithm and compare the results analytically with the existing algorithms. Recently another
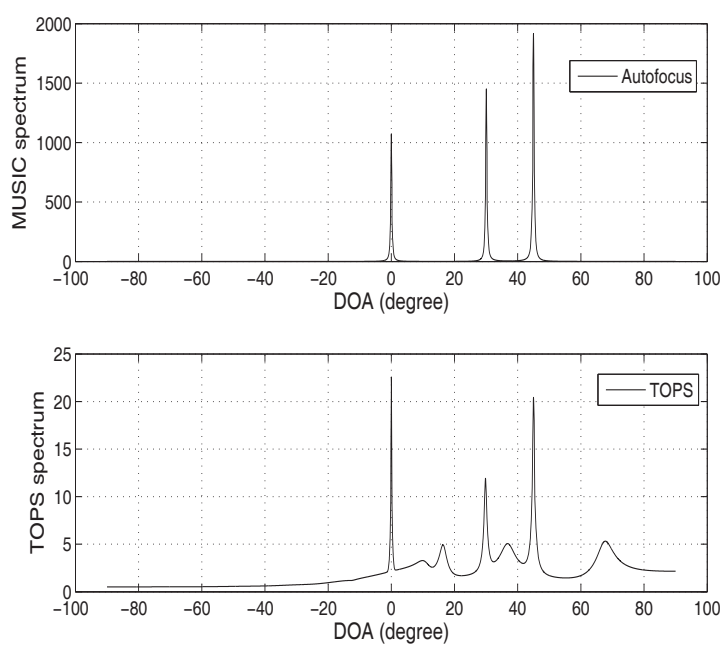

Fig. 3. Spatial spectrum for the three sources at $0^{\circ}, 30^{\circ}$ and $45^{\circ}$ using i) proposed autofocusing approach (top) and ii) TOPS (bottom) at $\mathrm{SNR}=0 \mathrm{~dB}$.

algorithm, namely TOFS [10], showed promising performance and in future we wish to compare the performance our method with it. Also, though in this paper, we concentrated on a passive array, we would like to extend this idea to the case of wideband DOA estimation using active arrays, e.g., the MIMO radar.

\section{REFERENCES}

[1] E. Di Claudio, and R. Parisi, "WAVES: weighted average of signal subspaces for robust wideband direction finding," IEEE Transactions on Signal Processing, vol.49, no.10, pp.2179-2191, Oct 2001.

[2] M. A. Doron and A. J. Weiss, "On focusing matrices for wide-band array processing," IEEE Transactions on Signal Processing, vol. 40, issue 6, pp. 1295-1302, Jun 1992.

[3] H. Hung and M. Kaveh, "Focussing matrices for coherent signal-subspace processing," IEEE Transactions on Acoustics, Speech and Signal Processing, vol.36, no.8, pp.1272-1281, Aug 1988.

[4] T. S. Lee, "Efficient wideband source localization using beamforming invariance technique," IEEE Transactions on Signal Processing, vol.42, no.6, pp.1376-1387, Jun 1994.

[5] R. Schmidt, "Multiple emitter location and signal parameter estimation," IEEE Transactions on Antennas and Propagation, vol. 34, no. 3, pp. 276280, 1986.

[6] D. N. Swingler, and J. Krolik, " Source location bias in the coherently focused high-resolution broad-band beamformer," IEEE Transactions on Acoustics, Speech and Signal processing, vol.37, no.1, pp.143-145, Jan 1989.

[7] H. Wang and M. Kaveh, "Coherent signal-subspace processing for the detection and estimation of angles of arrival of multiple wide-band sources," IEEE Transactions on Acoustics, Speech and Signal Processing, vol.33, no.4, pp. 823-831, Aug 1985.

[8] M. Wax, Tie-Jun Shan, and T. Kailath, "Spatio temporal spectral analysis by eigenstructure methods," IEEE Transactions on Acoustics, Speech and Signal Processing, vol.32, no.4, pp. 817-827, Aug 1984.

[9] Y. S. Yoon, L. M. Kaplan, and J. H. McClellan, "TOPS: new DOA estimator for wideband signals," IEEE Transactions on Signal Processing, vol.54, no.6, pp. 1977-1989, June 2006.

[10] H. Yu et al, "A New Method for Wideband DOA Estimation,"Int. Conf. on Wireless Communications, Networking, and Mobile Comuputing, pp. 598-601, 21-25 Sept. 2007. 